\section{Rare variants in dementia genes and Parkinson's disease}

European Journal of Human Genetics (2016) 24, 1661-1662; doi:10.1038/ejhg.2016.79; published online 22 June 2016

Parkinson's disease (PD) is a clinically heterogeneous disorder defined by the presence of cardinal motor symptoms. PD is frequently associated with progressive cognitive dysfunction, and longitudinal populationbased studies have indicated development of dementia in up to $80 \%$ of patients. ${ }^{1}$ The role of genetic factors in risk for cognitive decline in $\mathrm{PD}$ has been insufficiently studied. We, therefore, read with great interest the recently published article by Schulte et al. ${ }^{2}$, in which low-frequency and rare variants in seven genes involved in neurodegenerative dementia disorders were investigated by sequencing. They report more rare variants across seven genes in PD patients with dementia than in patients without dementia. Furthermore, they found that genetic variants in the $\beta$-amyloid precursor protein $(A P P)$ gene were more frequent in $\mathrm{PD}$ patients compared with both $\mathrm{AD}$ patients and healthy controls.

In an attempt to replicate these findings, we analyzed the sequencing data of the same seven dementia genes (APP, FUS, GRN, MAPT, PSEN1, PSEN2, and TARDBP) in a total of 517 Scandinavian PD patients and 230 healthy controls. All the participants provided written informed consent and the study was approved by the local ethics committee. Sequencing data from Norwegian PD patients $(n=387$; mean age at onset, 53 years) were generated by DNA pooling, HaloPlex target enrichment (Agilent Technologies, Santa Clara, CA, USA), and deep sequencing on a HiSeq2000 instrument (Illumina, San Diego, CA, USA), as described before. ${ }^{3}$ Sequencing of Swedish PD patients ( $n=130$; mean age at onset, 68 years) and Norwegian health controls ( $n=230$; mean age at inclusion, 62 years) was performed using an updated version of HaloPlex, without affecting the total coverage and depth of the sequenced regions. The ages of patients and controls were similar, in total, as the Norwegian PD patient group was younger than the controls and the Swedish older than the controls. Regarding our filtering process, we excluded from further analyses non-exonic variants except canonical splice sites, synonymous variants, and variants with frequency above 0.05 in the Exome Variant Server (http://evs.gs.washington.edu/EVS/), $1000 \mathrm{~g}$ (http://www.1000genomes. org) or an in-house database of 176 Norwegian exomes. Furthermore, variants were examined by visual inspection of the sequence alignment/ map format files to remove sequencing errors. Finally, to include only variants with predicted functional effects, the variants having a Combined Annotation Dependent Depletion score (CADD) ${ }^{4}$ above a cutoff value of 10 were retained.

We identified a total of 33 rare and low-frequency ( $<5 \% \mathrm{MAF}$ ) variants in the seven analyzed genes (Supplementary Table 1, all variants have been submitted to www.lovd.nl). 40/517 PD patients $(7.7 \%)$ and 23/230 controls (10\%) carried rare and low-frequency variants, indicating that there is no significant enrichment of dementia gene rare variants in $\operatorname{PD}\left(P=0.3, \chi^{2}\right.$-test $)$. By excluding the last filtering criteria - the CADD score - our results remained nonsignificant. Besides reporting an increased mutation burden in $\mathrm{PD}$ with dementia, Schulte et al. also reported that the APP rs140304729 variant is associated with PD. We identified $3 / 517$ patients (aged 47, 66, and 75 years) and 2/230 controls (aged 48 and 63 years) with rs 14030729 $\left(P=0.6, \chi^{2}\right.$-test $)$. We were, therefore, not able to replicate the association in our population, although we acknowledge that the power of our sample is moderate with $74 \%$ at $\alpha=0.05$, given a minor allele frequency of $0.3 \%$.

We also performed burden tests to analyze the load of rare variants in $A P P$. No excess of rare variants was found, as $A P P$ variants were identified in $13 / 517 \mathrm{PD}$ patients and $6 / 230$ controls $\left(P=0.9, \chi^{2}\right.$-test). The average age of controls with $A P P$ variants was 63.5 years. Similarly, burden tests remained nonsignificant for the other six genes (Supplementary Table 1). Usual burden tests are based on the principle of collapsing variants without assessing the directionality of the variants. However, genes do not only contain unidirectional variants; there can be risk, protective, and neutral variants. Interestingly, the APP gene is known to carry both disease-causing as well as protective variants for $\mathrm{AD} .^{5,6}$ We therefore performed the Sequence Kernel Association Test (SKAT), where neither directionality nor magnitudes of the associations are assumed a priori but instead estimated from the data. ${ }^{7}$ We used the SKAT test implemented in the SCORE-Seq (v5.3) program (http://www.bios.unc.edu/ lin/software/ SCORE/) using default settings. The test indicated no association for six of the dementia genes (Supplementary Table 1), but revealed a nominal significant $P$-value for MAPT (0.009), which is not significant after Bonferroni correction for analyses of seven genes. We found several novel rare variants with a high $\mathrm{CADD}^{4}$ score (Supplementary Table 1). CADD is a tool to score the deleteriousness of single nucleotide variants as well as small insertions and deletions. Thus, we cannot exclude that some of the identified rare variants in our study are of functional importance, but downstream functional analyses would be needed to test their possible role in affecting the normal function. None of the APP variants or any of the other dementia gene variants identified in our study have previously been reported to affect the protein function. ${ }^{8}$ However, we identified a proven protective variant of $\mathrm{AD}, \mathrm{p} .(\mathrm{A} 673 \mathrm{~T})^{9}$ in $2 / 517 \mathrm{PD}$ cases and $1 / 230$ controls. Of note, this variant has been found more frequently in Icelandic and Scandinavian populations as compared with other populations. ${ }^{5}$

Schulte et al. reported an increased number of dementia gene variants in PD with dementia compared with PD without dementia. We could not directly study this, as phenotypic data on cognitive function were only available in a minority of our samples. However, we would argue that it is problematic to dichotomize PD into two distinct groups: PD with and without dementia. Ageing is the most important risk factor for dementia in $\mathrm{PD}$, and the presence of dementia depends crucially on the timing of clinical assessment in the individual patient. It is in our view probably more useful to correlate risk factors with measures of cognitive function at certain time points in the course of the disease.

Onset and severity of cognitive deficits in PD is highly variable, and the biological basis for this variability is largely unknown. Previous studies have identified associations with coding and non-coding variants in SNCA, MAPT, APOE, and GBA - all known genetic risk factors for development of neurodegenerative disorders. ${ }^{10} A P P$, in our opinion, should not yet be added to this list. However, further 
studies of the biological basis of cognitive decline in PD and other neurodegenerative disorders should be highly encouraged.

\section{CONFLICT OF INTEREST}

The authors declare no conflict of interest.

\section{ACKNOWLEDGEMENTS}

We thank all individuals participating in the studies. Zafar Iqbal and Lasse Pihlstrøm have been supported by grants from South-Eastern Norway Regional Health Authority. Mathias Toft and Aina Rengmark have received funding from the Research Council of Norway. Lars Forsgren has been supported by a grant from the Swedish Medical Research Council.

Zafar Iqbal $^{1}$, Lasse Pihlstrøm ${ }^{1,2}$, Aina Rengmark ${ }^{1}$, Sandra Pilar Henriksen ${ }^{1}$, Jan Linder ${ }^{3}$, Lars Forsgren ${ }^{3}$ and

Mathias Toft ${ }^{1}$

${ }^{1}$ Department of Neurology, Oslo University Hospital, Oslo, Norway;

${ }^{2}$ Faculty of Medicine, University of Oslo, Oslo, Norway;

${ }^{3}$ Department of Pharmacology and Clinical Neuroscience, Umeå University, Umeå, Sweden E-mail: iqbal.z.phd@gmail.com
1 Hely MA, Reid WG, Adena MA, Halliday GM, Morris JG: The Sydney multicenter study of Parkinson's disease: the inevitability of dementia at 20 years. Mov Disord 2008; 23: 837-844.

2 Schulte EC, Fukumori A, Mollenhauer B et al: Rare variants in beta-Amyloid precursor protein (APP) and Parkinson's disease. Eur J Hum Genet 2015; 23: 1328-1333.

3 Pihlstrom L, Rengmark A, Bjornara KAToft M: Effective variant detection by targeted deep sequencing of DNA pools: an example from Parkinson's disease. Ann Hum Genet 2014; 78: 243-252.

4 Kircher M, Witten DM, Jain $\mathrm{P}$ et al: A general framework for estimating the relative pathogenicity of human genetic variants. Nat Genet 2014; 46: 310-315.

5 Jonsson T, Atwal JK, Steinberg $\mathrm{S}$ et al: A mutation in APP protects against Alzheimer's disease and age-related cognitive decline. Nature 2012; 488: 96-99.

6 Maloney JA, Bainbridge T, Gustafson A et al: Molecular mechanisms of Alzheimer disease protection by the A673T allele of amyloid precursor protein. J Biol Chem 2014; 289: 30990-31000.

7 Wu MC, Lee S, Cai T et al: Rare-variant association testing for sequencing data with the sequence kernel association test. Am J Hum Genet 2011; 89: 82-93.

8 Cruts M, Theuns J, Van Broeckhoven C: Locus-specific mutation databases for neurodegenerative brain diseases. Hum Mutat 2012; 33: 1340-1344.

9 Benilova I, Gallardo R, Ungureanu AA et al: The Alzheimer disease protective mutation A2T modulates kinetic and thermodynamic properties of amyloid-beta (Abeta) aggregation. J Biol Chem 2014; 289: 30977-30989.

10 Romo-Gutierrez D, Yescas P, Lopez-Lopez M, Boll MC: Genetic factors associated with dementia in Parkinson's disease (PD). Gac Med Mex 2015; 151: 110-118.

Supplementary Information accompanies this paper on European Journal of Human Genetics website (http://www.nature.com/ejhg) 